\title{
基于显著增强分层双线性池化网络的细粒度图像分类
}

\author{
陈珺莹，陈莹* \\ (江南大学轻工过程先进控制教育部重点实验室 无锡 214122) \\ (chenying@jiangnan.edu.cn)
}

\begin{abstract}
摘 要：分层双线性池化网络考虑了中间卷积层的特征交互，对细粒度图像起到了良好的分类效果，但它对一幅图 像包括无关背景在内的所有区域激活都进行了特征交互，会影响分类性能. 针对该问题，提出一种显著增强的分层 双线性池化方法. 该方法在分层双线性池化网络的基础上, 结合显著性检测网络生成注意力图, 使用注意力图与特 征提取网络进行交互实现对显著区域的信息增强，减少了背景等无关信息的影响，提高了分类性能. 在 3 个常用的细 粒度图像数据集 CUB-200-2011, Stanford Cars 和 FGVC-Aircraft 上均进行了实验，分类准确率分别为 $86.5 \%, 92.9 \%$ 和 $90.8 \%$ ，与当前其他主流方法相比，取得了良好的分类效果.
\end{abstract}

关键词：细粒度图像分类; 显著性检测; 区域信息增强; 分层双线性池化

中图法分类号: TP391.41 DOI: 10.3724/SP.J.1089.2021.18399

\section{Saliency Enhanced Hierarchical Bilinear Pooling for Fine-Grained Classification}

\author{
Chen Junying and Chen Ying* \\ (Key Laboratory of Advanced Process Control for Light Industry (Ministry of Education), Jiangnan University, Wuxi 214122)
}

\begin{abstract}
Hierarchical bilinear pooling network considers the feature interaction in middle convolutional layers and works well in the classification of fine-grained images. However, it carries out feature interaction on all activated image regions including irrelevant background, which affects the classification performance. To address this problem, a saliency enhanced hierarchical bilinear pooling method is proposed, which combines with the saliency detection network to generate an attention map, and uses the attention map to interact with the feature extraction network to enhance the information of the salient regions. As the result, it can reduce the impact of background and other irrelevant information, and improve the classification performance. The classification accuracy on three commonly used fine-grained image datasets CUB-200-2011, Stanford Cars and FGVC-Aircraft is $86.5 \%, 92.9 \%$ and $90.8 \%$, respectively, which is excellent compared with other mainstream methods.
\end{abstract}

Key words: fine-grained classification; saliency detection; regional information enhancement; hierarchical bilinear pooling

细粒度图像分类是近年来计算机视觉领域的 一个研究热点, 其目的是对粗粒度的大类进行更 加精细的子类划分. 随着深度学习的发展，许多计 算机视觉任务取得了不错的进展, 但是细粒度图
像分类仍然面临较大的困难. 其难点有 2 点: (1) 同一个子类中的物体之间存在个体的差异, 并且 还受到遮挡、尺度、姿态等的影响, 造成图像中的 外观差异可能会很大; (2) 同一个大类下不同的子

收稿日期: 2020-05-22; 修回日期: 2020-11-13. 基金项目：国家自然科学基金(61573168). 陈珺莹(1996一), 女, 硕士研究生, 主 要研究方向为计算机视觉; 陈莹(1976一), 女, 博士, 教授, 博士生导师, CCF 会员, 论文通讯作者, 主要研究方向为计算机视觉、信 息融合. 
类物体可能具有相似的外观和组成结构, 只存在 细微的差异, 且细节特征不易捕捉.

针对细粒度图像分类的难点, 往往需要定位 到图像中起区分作用的微小区域. 很多方法使用 额外人工标注来对这些微小区域做信息增强，即 基于强监督信息的分类方法. 然而，由于人工标注 信息的获取代价十分昂贵，并且人工标定的特定 的标注框或标注点并非最适合模型分类的区域， 因此基于弱监督信息的方法逐渐成为主流. Liu 等 ${ }^{[1]}$ 提出过滤蒸馏学习注意力模型, 用于增强细粒度 视觉分类中的区域注意力. Zheng 等 ${ }^{[2]}$ 提出三线注 意力采样网络, 利用三线注意力模块模型化通道 间的关系产生注意力图, 基于注意力的采样器将 关注区域通过高分辨率显示. Zhang 等 ${ }^{[3]}$ 提出一种 基于空间显著性提取的细粒度图像分类方法，由 图像的显著性信息获得物体的位置, 将物体裁剪 出来, 忽略掉背景的影响, 提高了分类性能. 除此 之外，对高阶特征编码的方式也取得了良好的分 类效果. Lin 等 ${ }^{[4]}$ 提出双线性卷积神经网络(bilinear convolutional neural networks, BCNN), 通过对最 后一个卷积层的输出特征进行外积操作, 以平移 不变的方式，对局部对级特征交互进行建模，取得 了优秀的性能. $\mathrm{Yu}$ 等 ${ }^{[5]}$ 在 $\mathrm{BCNN}$ 的基础上，提出分 层双线性池化(hierarchical bilinear pooling, HBP), HBP 网络对中间卷积层的特征也进行了交互, 提 高了分类性能.

虽然 HBP 有效地提高了分类准确率，但是它 对一幅图像的所有区域都进行了特征交互，包括 了一些无关的背景或不相干的物体区域，这些区 域会破坏特征交互，从而影响分类性能. 所以，需 要对这些无关的背景区域进行过滤，增强对感兴 趣区域的关注.

针对上述问题，本文提出了显著增强分层双 线性池化(saliency enhanced HBP, SE-HBP)网络. 显著性检测可以获取一幅图像中的感兴趣区域, 与文献[3]不同，本文并不对显著区域进行裁剪， 而是通过显著性检测得到一幅图像的显著性特征, 在此基础上得到注意力图; 再将注意力图与特征提 取网络中的特征进行乘积融合实现显著区域的信息 增强, 然后进行后续的特征提取及增强特征 HBP 操 作. 本文分别在 3 个常用的细粒度图像数据集 CUB-200-2011 ${ }^{[6]}$, Stanford Cars ${ }^{[7]}$ 和 FGVC-Aircraft ${ }^{[8]}$ 上进行了实验，实验结果表明，本文方法取得了较 好的分类性能，证明了显著性增强的有效性.

\section{1 本文方法}

本文基于显著性增强改进 HBP，具体而言，先 利用显著性检测网络得到一幅图像的显著性特征, 再由显著性特征生成注意力图，通过注意力图实 现显著区域的信息增强，以此增强对显著区域即 判别区域的关注，从而减少对背景等无关信息的 关注. 下面分别介绍 HBP 的问题所在以及 SE-HBP.

\subsection{HBP 及其问题分析}

$\mathrm{BCNN}^{[4]}$ 实现了端到端的细粒度图像分类; $\mathrm{Yu}$ 等 ${ }^{[5]}$ 指出, BCNN 只考虑了最后一个卷积层的特征 交互，忽视了卷积层之间的特征交互与细粒度特 征的学习是可以相互加强的, 所以提出 HBP 来捕 获层间的特征交互, 并集成了多个分层双线性特 征, 提高了特征的表示能力.

本文使用 ResNet-34 构建 HBP 网络, 对 ResNet-34 最后一组(即第 4 组)块(block)的 3 个特 征进行 HBP 操作, 这 3 个特征分别表示为 $\boldsymbol{X}_{4-1}$, $\boldsymbol{X}_{4-2}$ 和 $\boldsymbol{X}_{4-3}$, 如图 1 所示选取 2 幅图像, 对这 3 个特征激活进行了可视化. 图 1 中, 第 1 列是从数 据集中选取的图像，其他 3 列是 ResNet-34 最后一 组 block 的 3 个特征激活的热力图, 红色区域表示 网络更加关注的区域, HBP 模型对这 2 幅图像分类 错误，最后一列是错误类别中的示例图像.

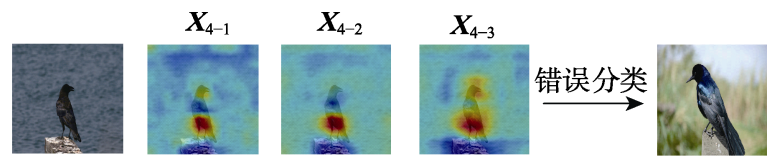
a. 鱼鸭误判为宽尾拟八哥

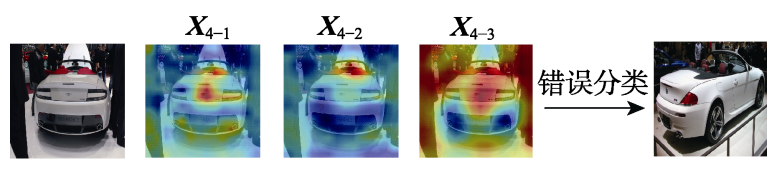

b. 阿斯顿马丁误判为宝马

图 1 最后一组 block 的 3 个特征激活的 热力图及错误分类的类别

图 1a 选取的是 CUB-200-2011 数据集中的一 幅类别为鱼鸭的鸟类图像，被错误分类成宽尾拟 八哥. 从特征激活的热力图可以看出，网络只关注 到了鱼鸭的头部和飞羽部位区域，这些区域与宽 尾拟八哥没有明显的不同; 鱼鸭的背部通常是纯 黑色的，而宽尾拟八哥的背部呈现亮蓝色或亮绿 色，但是 HBP 并没有关注到这部分区域，导致分 类错误. 图 $1 \mathrm{~b}$ 选取的是 Stanford Cars 数据集中的 一幅车辆图像, 是阿斯顿马丁敞篻车的车身尾部, 被错误分类为宝马敞篷车. 从阿斯顿马丁敞篷车 
的尾部来看, 其与宝马敞篷车的最大不同在于车 尾灯区域，而 HBP 没有关注到这部分区域，反而 关注到了无关的背景(车旁的人)，导致分类错误.

所以，对于 HBP 网络，一些感兴趣区域(即判 别区域)没有得到关注，而一些无关的背景或不相 关的区域在特征激活中有较高的响应, HBP 网络对 这些区域进行了特征交互, 影响了分类性能. 需要 考虑如何减少无关背景的影响、如何更好地关注感 兴趣区域，从而实现更好的特征交互. 显著性检测 网络可以获取到一幅图像中的感兴趣区域，针对 上述问题，本文结合显著性检测网络来增强对感 兴趣区域即判别区域的关注，减少背景等无关信 息的影响。

\subsection{SE-HBP}

为了更好地关注感兴趣区域，文献[9-11]均通 过物体标注框或部位标注点等额外人工标注对物 体整体或局部区域进行定位，实现区域信息的增
强; 但是这些方法依赖的人工标注成本较高, 在实 际应用中有局限性. 由于显著性检测可以模拟人 的视觉注意力机制, 提取图像中的感兴趣区域, 本 文结合显著性检测网络实现感兴趣区域的信息增 强. 即通过显著性检测网络得到的显著性特征, 以 注意力图的形式来增强感兴趣区域的信息，同时 减少背景等无关区域的信息.

整体的网络结构如图 2 所示, 分为显著性检 测、注意力生成以及增强特征 HBP 3 个部分. 首先 经过显著性检测网络获得显著性特征, 再由此得 到注意力图; 该注意力图的取值范围为 $0 \sim 1$. 对于 显著区域，即感兴趣区域，注意力图中相应区域的 值接近 1 ; 对于背景等无关区域, 注意力图中相应 区域的值很小. 采用 ResNet-34 构建 SE-HBP 网络, 将注意力图与 ResNet-34 第 3 组 block 最后输出的 特征进行乘积融合, 再进行后续的特征提取以及 增强特征 HBP 操作.

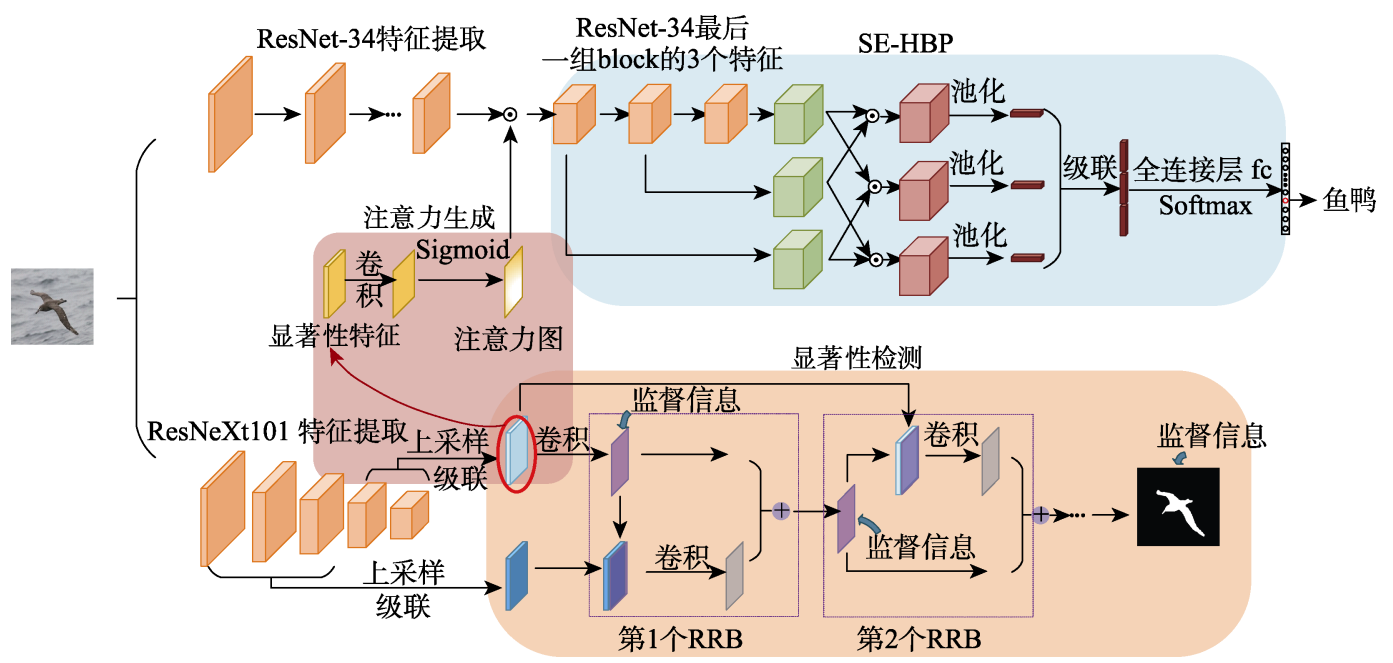

口特征提取层; $\square$ 投影层; $\square$ 交互层; $\square$ 显著图; $\square$ 残差; $\square$ 显著性特征; $\square$ 高层集成特征; $\square$ 低层集成特征

图 2 本文网络结构

\section{2 .1 显著性检测}

显著性检测旨在提取图像中的显著区域，即 感兴趣区域. 本文使用的显著性检测网络是循环 残差优化网络(recurrent residual refinement networks, $\left.\mathrm{R}^{3} \mathrm{Net}\right)^{[12]}$ ，其网络结构如图 2 的橙色区域所 示，它包含很多个残差优化块(residual refinement block, RRB). RRB 可以交替利用全卷积网络的低 层集成特征和高层集成特征来学习中间显著性预 测和真实值之间的残差，低层集成特征可以捕获 更多的显著性细节，高层集成特征可以减少中间 预测的非显著区域.

首先，通过特征提取网络(这里用 ResNeXt101)
生成一组特征图，其中包含了不同尺度的低层细 节和高级语义信息. 然后, 浅层特征通过集成生成 低层集成特征，深层特征聚合成高层集成特征，提 取特征后，从高层集成特征生成一个初始的显著 图，然后交替利用低层集成特征和高层集成特征 生成 RRB，逐步完善中间显著图. RRB 执行的具体 操作为: 将前一个循环得到的显著性预测图交替 地与低层或高层集成特征相连接, 经过卷积得到 当前循环的残差，再将残差与前一个循环的显著 性预测图相加，得到当前循环的显著性预测图.

\subsection{2 注意力生成}

在 $\mathrm{R}^{3} \mathrm{Net}$ 的训练过程中，高层集成特征首先在 
真实值的监督下生成一个初始的显著图，然后用 一系列的 RRB 对其进行完善. 所以，使用 $\mathrm{R}^{3} \mathrm{Net}^{[12]}$ 模型，采用其中的高层集成特征作为显著性特征. 对于低层集成特征，其在训练时不直接接受真实 值的监督，所以低层集成特征包含的显著性信息 不如高层集成特征的好. 本文在实验部分比较了 分别使用高层和低层集成特征作为显著性特征得 到的分类准确率，验证了高层集成特征比低层集 成特征更有效.

通过显著性检测网络 $\mathrm{R}^{3} \mathrm{Net}$ 得到显著性特征 $\boldsymbol{X}_{\text {sal }} \in \mathbb{R}^{h_{1} \times w_{1} \times c_{1}}$ ，其中， $h_{1}$ 为高， $w_{1}$ 为宽， $c_{1}$ 为通 道数; 再经过降维以及 Sigmoid 函数生成 1 通道的 注意力图 $\boldsymbol{X}_{\mathrm{att}} \in \mathbb{R}^{h_{1} \times w_{1} \times 1}$.

\subsection{3 增强特征 HBP}

HBP 网络(网络结构如图 2 中的蓝色区域所示) 是在因式分解双线性池化 (factorized bilinear pooling, FBP $)^{[13]}$ 网络基础上改进的. 假设一幅图像经 过卷积神经网络进行特征提取后得到的输出特征 为 $\boldsymbol{X} \in \mathbb{R}^{h \times w \times c}$. 其中, $h$ 为高， $w$ 为宽， $c$ 为通道 数; 输出特征可以看做由 $c$ 个大小为 $h \times w$ 的二维 特征组成，用 $\boldsymbol{X}^{\vdots ;, k} \in \mathbb{R}^{h \times w}$ 表示第 $k$ 个通道的特征 图; 也可以看做由 $h \times w$ 个 $c$ 维描述符组成，用 $\boldsymbol{X}^{i, j,:} \in \mathbb{R}^{c}$ 表示一个特定位置 $(i, j)$ 上的描述符; 其 中, $i \in\{1, \cdots, h\}, j \in\{1, \cdots, w\}$. FBP 模型定义为每个 空间位置的低秩外积操作, 即 $\boldsymbol{o}_{\mathrm{FBP}}=\boldsymbol{P}^{\mathrm{T}}\left(\boldsymbol{U}^{\mathrm{T}} \boldsymbol{x} \odot \boldsymbol{V}^{\mathrm{T}} \boldsymbol{x}\right)$. 其中, $\boldsymbol{x}=\boldsymbol{X}^{i, j, ;}$ 为特定位 置 $(i, j)$ 的 $c$ 维描述符; $\boldsymbol{P} \in \mathbb{R}^{d \times d^{\prime}}$ 为分类矩阵 $; d$ 为特征向量的维数; $d^{\prime}$ 为图像的类别数; $\boldsymbol{U} \in \mathbb{R}^{c \times d}$ 和 $\boldsymbol{V} \in \mathbb{R}^{c \times d}$ 为投影矩阵； $\odot$ 表示对应元 素相乘; $\boldsymbol{o}_{\mathrm{FBP}} \in \mathbb{R}^{d^{\prime}}$ 为输出向量.

与文献 [14] 的基础网络一致，本文使用 ResNet-34 构建 SE-HBP 网络. 假设图像经过 ResNet-34 第 3 组 block 后得到的特征为 $\boldsymbol{X}_{3} \in \mathbb{R}^{h_{1} \times w_{1} \times c_{2}}$; 其中， $h_{1}$ 为高， $w_{1}$ 为宽， $c_{2}$ 为通 道数. 因为经过 ResNet-34 第 3 组 block 后的特征 与经过 $R^{3} \mathrm{Net}$ 得到的显著性特征仅通道数不同，特 征的高与宽是相同的, 所以不需要对注意力图的 高和宽进行处理. 将注意力图与 ResNet-34 第 3 组 block 得到的特征相乘, 得到 $\boldsymbol{X}_{3}{ }^{\prime}=\boldsymbol{X}_{3} \odot \boldsymbol{X}_{\text {att }}$. 对 $\boldsymbol{X}_{3}^{\prime}$ 进行后续的特征提取，经过 ResNet-34 最后一 组 block 得到的 3 个特征分别为 $\boldsymbol{X}_{4-1} \in \mathbb{R}^{h \times w \times c}$, $\boldsymbol{X}_{4-2} \in \mathbb{R}^{h \times w \times c}$ 和 $\boldsymbol{X}_{4-3} \in \mathbb{R}^{h \times w \times c}$ ，它们都融人了显 著性特征，实现了显著增强. 这 3 个显著增强后的
卷积特征通过层叠多个 HBP 模块进行合并, 利用 了层间的特征交互关系，增强了特征的描述能力. 对于每个空间位置, SE-HBP 模型的输出为

$$
\begin{aligned}
& \boldsymbol{o}_{\text {SE-HBP }}=\boldsymbol{P}^{\mathrm{T}} \operatorname{concat}\left(\boldsymbol{U}^{\mathrm{T}} \boldsymbol{x}_{4-1} \odot \boldsymbol{V}^{\mathrm{T}} \boldsymbol{x}_{4-2}, \boldsymbol{U}^{\mathrm{T}} \boldsymbol{x}_{4-1} \odot\right. \\
& \left.\boldsymbol{S}^{\mathrm{T}} \boldsymbol{x}_{4-3}, \boldsymbol{V}^{\mathrm{T}} \boldsymbol{x}_{4-2} \odot \boldsymbol{S}^{\mathrm{T}} \boldsymbol{x}_{4-3}\right) .
\end{aligned}
$$

其中, $\boldsymbol{x}_{4-1}=\boldsymbol{X}_{4-1}^{i, j,:}, \boldsymbol{x}_{4-2}=\boldsymbol{X}_{4-2}{ }^{i, j,:}, \boldsymbol{x}_{4-3}=\boldsymbol{X}_{4-3}{ }^{i, j,}$ 分别为 3 个卷积特征的特征描述符; $\boldsymbol{U} \in \mathbb{R}^{c \times d}$, $\boldsymbol{V} \in \mathbb{R}^{c \times d} ， \boldsymbol{S} \in \mathbb{R}^{c \times d}$ 分别为 3 个投影矩阵; $\boldsymbol{P} \in \mathbb{R}^{3 d \times d^{\prime}}$ 为分类矩阵，在实际中分类采用全连接 层(fully connected layer, fc)来实现， $d^{\prime}$ 为图像的类 别数; $\operatorname{concat}()$ 为通道级联操作; $\boldsymbol{o}_{\mathrm{SE}-\mathrm{HBP}} \in \mathbb{R}^{d^{\prime}}$ 为 输出向量.

得到输出向量之后，使用 Softmax 函数将分类 预测的输出标准化, 得到输人图像归属于各个类 别的概率分布; 然后使用交叉熵作为损失函数计 算预测分类与真实结果之间的相似度. 在实验部 分, 本文还比较了将注意力图与 $\boldsymbol{X}_{4-1}$ 以及 $\boldsymbol{X}_{4-2}$ 相 乘的分类准确率, 验证当注意力图与第 3 组 block 的输出特征 $\boldsymbol{X}_{3}$ 相乘时能获得最优的识别效果.

\section{2 实验及结果分析}

\section{1 数据集}

在 3 个常用的细粒度图像数据集上进行了实 验, 分别是 CUB-200-2011, Stanford Cars 和 FGVC-Aircraft. CUB-200-2011 是加利福尼亚理工 学院创建的鸟类数据集, 包括了 11788 幅鸟类图 像, 共有 200 个子类; Stanford Cars 数据集包含了 196 种车型, 共有 16185 幅图像; FGVC-Aircraft 数 据集包含 10000 幅飞机图像，共 100 个子类. 每个 数据集的详细信息如表 1 所示.

表 1 数据集信息

\begin{tabular}{lccc}
\hline \multicolumn{1}{c}{ 数据集 } & 类别数 & 训练集/幅 & 测试集/幅 \\
\hline CUB-200-2011 & 200 & 5994 & 5794 \\
Stanford Cars & 196 & 8144 & 8041 \\
FGVC-Aircraft & 100 & 6667 & 3333 \\
\hline
\end{tabular}

\section{2 参数设置}

实验采用了开源 Linux 内核的 Ubuntu14.04 桌面 应用系统, PyTorch 深度学习框架, Python 编程语言.

在训练 $\mathrm{R}^{3} \mathrm{Net}$ 时, 使用了在 ImageNet 数据集 ${ }^{[15]}$ 上预训练的 ResNeXt101 初始化特征提取网络; 然 后将 ResNeXt101 的前 3 层特征聚合构成低层集成 特征，后 2 层特征聚合构成高层集成特征，权衡时 
间性能以及检测精度，使用了 6 个 RRB. 在 MSRA10K 数据集 ${ }^{[16]}$ 上进行训练, 每个循环中的显 著性预测都与真实值计算交叉熵损失，这些交叉 熵损失的和构成总的损失函数. 训练过程使用了 随机梯度下降 (stochastic gradient descent, SGD), 权重衰减(weight decay)为 $5 \times 10^{-4}$, 动量 (momentum) 为 0.9 , 学习率初始化为 0.001 , 使用了 poly 策略 ${ }^{[17]}$ 调整学习率, 在 6000 次迭代后训练完成.

与文献[14]一致, 使用在 ImageNet 数据集上 预训练的 ResNet-34 构建 SE-HBP 网络, 去除 ResNet-34 最后的全连接层. 训练过程分为 2 步:

Step1. 只训练在原始 ResNet-34 基础上新增加的层.

Step2. 微调整个网络. 显著性检测网络 $\mathrm{R}^{3} \mathrm{Net}$ 的权 重始终固定。

对于图进行预处理，数据集 CUB-200-2011, Stanford Cars 和 FGVC-Aircraft 的图像像素大小分 别固定为 $600 \times 600 ， 500 \times 500$ 以及 $500 \times 480$, 将图 像裁剪到 $448 \times 448$, 在训练过程中使用了随机裁 剪, 测试过程中使用的是中心裁剪. 对于投影层的 维数, 文献[5]验证了投影到 8192 维效果最好, 文
献[14]也是投影到 8192 维, 所以，本文将 ResNet-34 最后一组 block 得到的 3 个特征都由 512 维投影到 8192 维. 使用的优化器为 SGD, weight decay 为 $1 \times 10^{-5}$, momentum 为 0.9 . 第 1 步训练中学习率初 始化为 1 , 第 2 步训练中学习率初始化为 0.01 , 每 隔 40 个周期学习率下降 $90 \%$.

\section{3 显著增强的效果}

从上述 3 个细粒度图像数据集中选取一些图 像, 比较 SE-HBP 以及 HBP 网络的特征, 可视化结 果如图 3 所示. 图 3 前 4 列含义与图 1 中前 4 列含 义相同，最后 3 列是进行了显著增强的特征激活的 热力图.

图 3a 选取的是 CUB-200-2011 中的 2 幅图像, 上下 2 行分别是丽色凤头燕鸥和鱼鸭的热力图, HBP 网络关注到丽色风头燕鸥图像中的树干、海 水等无关背景，而显著增强后，对无关背景的关注 减弱. 对于鱼鸭, HBP 网络将其错误分类成了宽尾 拟八哥，是因为 HBP 网络没有关注到鱼鸭和宽尾 拟八哥起区分作用的背部，而显著增强后的网络 可以关注到背部. 图 $3 \mathrm{~b}$ 是本田雅阁轿车和阿斯顿

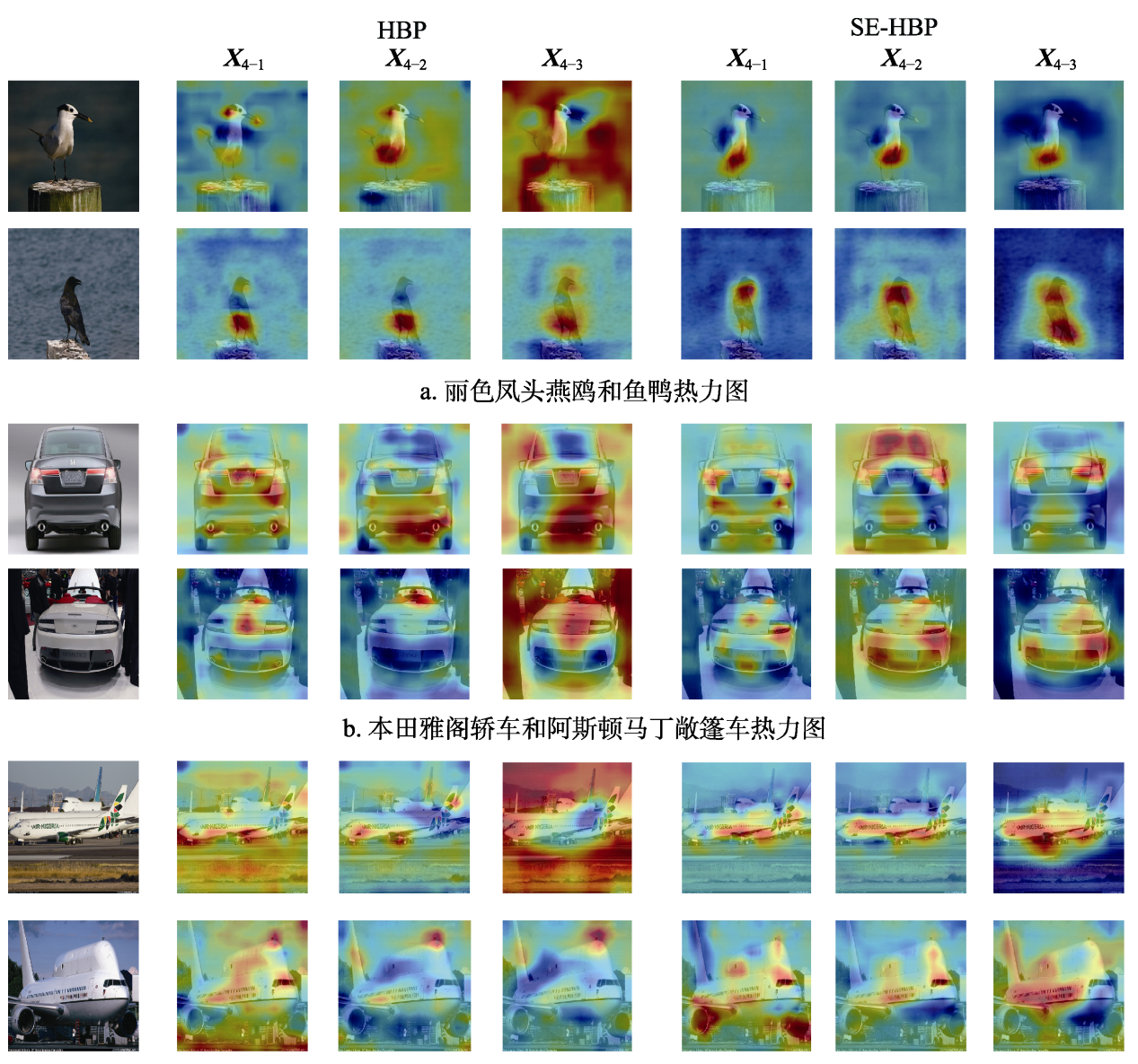

c. 波音737-400和波音767-200热力图

图 3 热力图对比 
马丁敞篷车的热力图, HBP 网络关注到了本田雅阁 轿车旁的无关背景, 而 SE-HBP 可以关注到更多的 车身部分且过滤掉了无关背景, HBP 网络将阿斯顿 马丁敞篷车误判成宝马敞篷车, 改进后的网络关 注到了阿斯顿马丁敞篷车的车尾灯区域, 这是与 宝马敞篷车尾部最大的不同, 并且对旁边的行人 等无关背景的关注减弱. 图 3c 是机型分别为波音 737-400 和波音 767-200 的 2 架飞机, SE-HBP 关注 到波音 737-400 更多的机身部分，忽略了对天空、 地面等无关背景的关注; 对于波音 767-200, SE-HBP也关注到其引擎以及机顶等起区分作用的 关键区域. HBP 网络会关注到一些无关的背景区 域，对这些区域进行特征交互会影响分类性能，且 其关注到的局部区域可能不是最具判别性的区域, 而 SE-HBP 网络对这几幅图像均可以分类正确，其 可以关注到 HBP 网络没有关注到的起区分作用的 关键区域，并且可以减弱对背景或不相关区域的 关注，从而获得更好的分类性能.

\section{4 分类性能}

\section{4 .1 卷积层选择}

本文首先通过显著性特征得到注意力图，然 后将注意力图与 ResNet-34 第 3 组 block 后的特征 进行点乘加权增强处理, 经过 ResNet-34 第 3 组 block 后的特征表示为 $\boldsymbol{X}_{3}$, 再将相乘后的特征送人 ResNet-34 最后一组 block 进行特征提取. ResNet-34 最后一组 block 共有 3 个残差块, 经过这 3 个残差块得到的特征分别表示为 $\boldsymbol{X}_{4-1}, \boldsymbol{X}_{4-2}$ 和 $\boldsymbol{X}_{4-3}$. 本文在 CUB-200-2011，Stanford Cars, FGVC-Aircraft 这 3 个数据集上, 将注意力图与不同 卷积层的特征相乘，对比其分类准确率，结果如表 2 所示.

表 2 与不同卷积层相乘的分类准确率对比 \%

\begin{tabular}{lccc}
\hline \multicolumn{1}{c}{ 数据集 } & $\boldsymbol{X}_{3}$ & $\boldsymbol{X}_{4-1}$ & $\boldsymbol{X}_{4-2}$ \\
\hline CUB-200-2011 & 86.5 & 86.0 & 85.9 \\
Stanford Cars & 92.9 & 92.5 & 92.2 \\
FGVC-Aircraft & 90.8 & 90.6 & 90.3 \\
\hline
\end{tabular}

注意力图与特征 $\boldsymbol{X}_{4-1}$ 相乘时, 将相乘后的特 征送人 ResNet-34 最后一组 block 的后 2 个残差块 继续进行特征提取, 再将相乘前的特征与后 2 个残 差块的输出特征进行双线性池化操作. 同理, 注意 力图与特征 $X_{4-2}$ 相乘时, 先经过了最后一组 block 的前 2 个残差块得到 $\boldsymbol{X}_{4-1}$ 和 $\boldsymbol{X}_{4-2}$, 再将注意力图 与 $\boldsymbol{X}_{4-2}$ 相乘, 然后相乘后的特征送人最后一个残 差块, 最后将相乘前的特征与前后 2 个残差块的输
出进行双线性池化操作. 注意力图与 $X_{4-1}, X_{4-2}$ 相乘时, 高和宽为 $X_{4-1}, X_{4-2}$ 的 2 倍, 所以需要 将注意力图的高和宽调整为原来的 $1 / 2$.

由表 2 可知, 在 3 个数据集上, 当注意力图与 ResNet-34 第 3 组 block 最后输出的特征 $\boldsymbol{X}_{3}$ 相乘时, 均能得到最高的分类准确率. 这是因为当注意力 图与 $X_{3}$ 相乘时, 相乘后的特征送人 ResNet-34 最 后一组 block, 得到的 $X_{4-1}, X_{4-2}, X_{4-3}$ 都融人了 显著性特征; 而与 $\boldsymbol{X}_{4-1}$ 相乘时，只有 $\boldsymbol{X}_{4-2}$ 和 $\boldsymbol{X}_{4-3}$ 融人了显著性特征, 与 $X_{4-2}$ 相乘时, 只有 $X_{4-3}$ 进 行了显著增强. 以下实验都建立在注意力图与 $X_{3}$ 相乘的基础上.

\subsection{2 显著性特征的选择}

在 $R^{3} \mathrm{Net}$ 中，首先是高层集成特征在真实值的 监督下生成一个初始的显著图, 然后 RRB 交替地 利用高层和低层集成特征对其进行完善. 在 CUB-200-2011, Stanford Cars, FGVC-Aircraft 这 3 个数据集上, 比较了使用高层和低层集成特征分 别作为显著性特征生成注意力图的分类准确率, 如表 3 所示.

表 3 高低层集成特征作为显著性特征的 分类准确率对比

\begin{tabular}{lcc}
\hline 数据集 & 高层集成特征 & 低层集成特征 \\
\hline CUB-200-2011 & 86.5 & 86.3 \\
Stanford Cars & 92.9 & 92.5 \\
FGVC-Aircraft & 90.8 & 90.4 \\
\hline
\end{tabular}

表 3 中, 显著性特征生成的注意力图均是与 ResNet-34 第 3 组 block 最后输出的特征 $\boldsymbol{X}_{3}$ 相乘. 高层集成特征生成的注意力图的高和宽与 $X_{3}$ 的高 和宽一致, 所以不需要作额外的处理; 而低层集成 特征生成的注意力图的高和宽是 $\boldsymbol{X}_{3}$ 的 4 倍, 需要 对其作下采样处理. 实验结果显示, 在 3 个数据集 上, 高层集成特征作为显著性特征得到的分类准 确率均比低层集成特征作为显著性特征高.

为进一步验证高层集成特征的分类优势，利 用 Python 的 Matplotlib 库分别对高层和低层集成 特征生成的注意力图进行可视化, 结果如图 4 所 示. 图 4 中第 1 列是选自 3 个数据集的图像，第 2 列和第 3 列分别是高层和低层集成特征生成的注 意力图，显示了不同颜色所对应值.

由图 4 可知, 高层集成特征生成的注意力图能 够更好地过滤掉背景等无关信息, 更好地保留了 

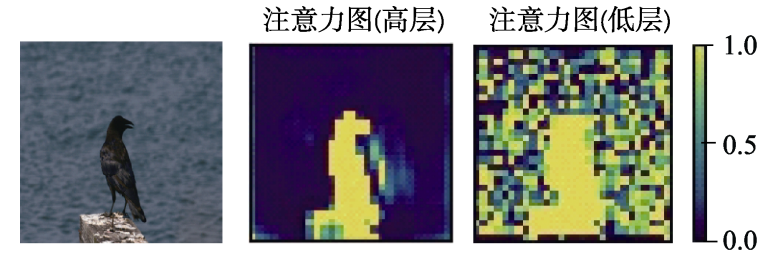

a. 鱼鸭 注意力图(高层)
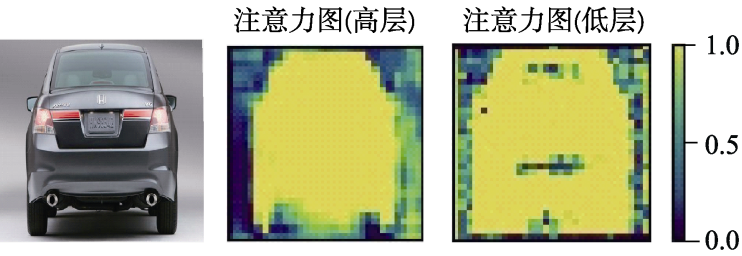

b. 本田雅阁轿车

注意力图(高层)
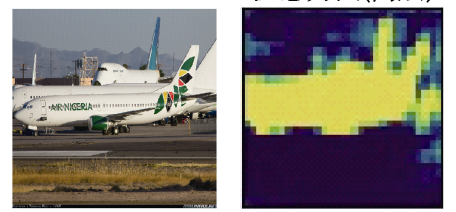

注意力图(低层)

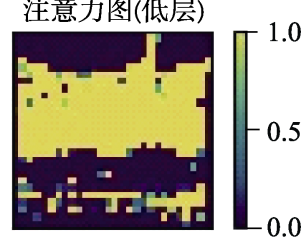

c. 波音 $737-400$

图 4 注意力图比较

感兴趣区域，以下实验的显著性特征均采用高层 集成特征.

\section{4 .3 与基线方法比较}

本文的基线方法是使用 ResNet-34 重新构建的 HBP 网络(HBP-RNet) ${ }^{[14]}$, 本文在 CUB-200-2011, Stanford Cars 和 FGVC-Aircraft 这 3 个数据集上都 进行了实验, 并比较了 SE-HBP 网络和基线网络的
准确率, 结果如图 5 所示.

由图 5 可知, SE-HBP 在 CUB-200-2011, Stanford Cars 和 FGVC-Aircraft 这 3 个数据集上的分类 准确率分别为 $86.5 \%, 92.9 \%$ 和 $90.8 \%$, 基线方法的 分类准确率分别为 $85.8 \%, 92.2 \%$ 和 $90.2 \%$, 可以看 出, 本文方法在这 3 个数据集上的分类准确率都优 于基线方法.

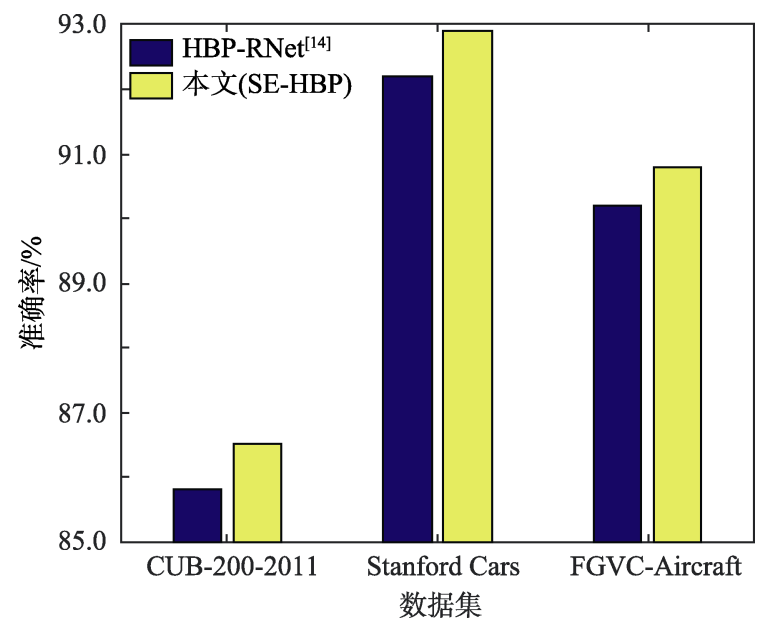

图 5 本文方法与基线方法准确率对比

\subsection{4 与其他方法比较}

表 4 列出了在 3 个细粒度图像数据集上的分类 实验结果, 并与相关方法做了比较. 本文方法只使 用了图像的类别标签，没有使用额外人工标注.

表 4 本文方法与相关方法的准确率对比

\begin{tabular}{|c|c|c|c|c|c|}
\hline 分类 & 方法 & 额外人工标注 & CUB-200-2011 & Stanford Cars & FGVC-Aircraft \\
\hline \multirow{2}{*}{ 基于部件且使用额外的人工标注 } & $\mathrm{SPDA}^{-\mathrm{CNN}^{[18]}}$ & $\sqrt{ }$ & 85.1 & & \\
\hline & $\mathrm{PC}-\mathrm{CNN}^{[19]}$ & $\sqrt{ }$ & 84.1 & & \\
\hline \multirow{3}{*}{ 基于部件只使用图像类别标签 } & $\mathrm{MA}-\mathrm{CNN}^{[20]}$ & & 86.5 & 92.8 & 89.9 \\
\hline & $\mathrm{RP}-\mathrm{CNN}^{[21]}$ & & 84.5 & 93.0 & 89.9 \\
\hline & $\mathrm{UPM}^{[22]}$ & & 85.4 & 92.3 & 90.0 \\
\hline \multirow{8}{*}{ 基于双线性网络及其改进 } & $\mathrm{BCNN}^{[4]}$ & & 84.1 & 91.3 & 84.1 \\
\hline & $\mathrm{CBP}^{[23]}$ & & 84.0 & & \\
\hline & $\mathrm{LRBP}^{[24]}$ & & 84.2 & 90.1 & 87.3 \\
\hline & $\mathrm{KP}^{[25]}$ & & 86.2 & 92.4 & 86.9 \\
\hline & $\mathrm{GP}^{[26]}$ & & 85.8 & 92.8 & 89.8 \\
\hline & HBP-RNet ${ }^{[14]}$ & & 85.8 & 92.2 & 90.2 \\
\hline & Dai 等 ${ }^{[27]}$ & & 85.3 & 92.4 & \\
\hline & SE-HBP & & 86.5 & 92.9 & 90.8 \\
\hline
\end{tabular}

注. 粗体表示精度最高.

表 4 中，基于部件且使用额外的人工标注的方 法, 分别是联合语义部件检测和提取的卷积神经 网络(unifying semantic part detection and abstraction convolutional neural networks, SPDA-CNN ${ }^{[18]}$, 以及
基于深度协同卷积网络的细粒度分类 (part-collaboration convolutional neural networks, PC$\mathrm{CNN})^{[19]}$, 后者在每个手工标注的部件上训练定制 的子网络. 
基于部件只使用图像类别标签的方法分别有 多注意力卷积神经网络(multi-attention convolutional neural networks, MA-CNN) ${ }^{[20]}$, 其利用通道分组子 网络生成多个部件; 随机部件定位模型 (random part localization convolutional neural networks, $\mathrm{RP}-\mathrm{CNN})^{[21]}$ 使用显著图提取前景物体; 部件挖掘 (unsupervised part mining, UPM) ${ }^{[22]}$ 方法利用模式 挖掘算法来获取判别区域。

还有一些基于双线性网络以及在其基础上改 进的方法, 如紧凑的双线性池化方法 (compact bilinear pooling, CBP) ${ }^{[23]}$ 、低秩双线性池化(low-rank bilinear pooling, LRBP) ${ }^{[24]}$ 、通用的池化框架(kernel pooling, KP) ${ }^{[25]}$ 、格拉斯曼池化(Grassmann pooling, $\mathrm{GP})^{[26]}, \mathrm{HBP}^{[5]}$ 和基于子类别相似性度量的双线性 池化 ${ }^{[27]}$ ，都是在 $\mathrm{BCNN}^{[4]}$ 的基础上改进的.

由表 4 结果可知, 本文方法(SE-HBP)在 3 个数 据集上都取得了较好的分类性能，都优于基线方 法(HBP-RNet)。注意，本文的基线方法为使用 ResNet-34 重新构建的 HBP 网络(HBP-RNet $)^{[14]}$, 因 为 ResNet-34 是一个轻量级的网络, 与构建 HBP 网络 ${ }^{[5]}$ 的 VGG-Net ${ }^{[28]}$ 相比, ResNet-34 的计算效率 更高. 由表 4 可知，本文方法比双线性网络以及在 其基础上改进方法的分类准确率都要高. 由此可 见, SE-HBP 可以实现对显著区域即感兴趣区域的 信息增强，减少背景等无关信息的影响，使网络关 注到更具判别性的区域，从而获得表述能力更好 的特征; 本文方法比使用了额外人工标注信息的 局部信息增强方法的准确率高，因为人工标注的 区域并非是最适合模型分类的区域，证明了本文 使用显著性检测网络可以获取到比人工标注更为 合理的感兴趣区域，从而获得良好的分类性能，同 时避免了使用成本较高的人工标注; 本文方法比 大多数不使用额外人工标注的局部信息增强的方 法的分类效果好，证明了本文使用显著增强来获 取感兴趣区域的做法可以与一些复杂的部件检测 方法达到类似的效果.

\section{3 结 语}

本文提出了适用于细粒度图像分类的 SE-HBP 网络，使用显著性检测网络得到图像的显著性特 征, 并将显著性特征以注意力图的形式与原来的 特征相结合，以此增强显著区域的信息，减少背景 等无关信息的影响, 实现网络的显著性增强. 本文 在 3 个常用的细粒度图像数据集上都进行了实验,
在 CUB-200-2011, Stanford Cars 和 FGVC-Aircraft 上分别取得了 $86.5 \%, 92.9 \%$ 和 $90.8 \%$ 的分类准确 率，均优于基线方法，与其他对比方法相比，取得 了具有竞争力的分类准确率，得到了良好的分类 性能，验证了本文方法的有效性.

\section{参考文献(References):}

[1] Liu C B, Xie H T, Zha Z J, et al. Filtration and distillation: enhancing region attention for fine-grained visual categorization[C] //Proceedings of the AAAI Conference on Artificial Intelligence. Palo Alto: AAAI Press, 2020: 11555-11562

[2] Zheng $\mathrm{H} \mathrm{L}, \mathrm{Fu} \mathrm{J}$ L, Zha Z J, et al. Looking for the devil in the details: learning trilinear attention sampling network for fine-grained image recognition[C] //Proceedings of the IEEE Conference on Computer Vision and Pattern Recognition. Los Alamitos: IEEE Computer Society Press, 2019: 5007-5016

[3] Zhang J T, Sun F W, Song J, et al. Fine-grained image classification via spatial saliency extraction[C] //Proceedings of the 17th IEEE International Conference on Machine Learning and Applications. Los Alamitos: IEEE Computer Society Press, 2018: 249-255

[4] Lin T Y, RoyChowdhury A, Maji S. Bilinear CNN models for fine-grained visual recognition[C] //Proceedings of the IEEE International Conference on Computer Vision. Los Alamitos: IEEE Computer Society Press, 2015: 1449-1457

[5] Yu C J, Zhao X Y, Zheng Q, et al. Hierarchical bilinear pooling for fine-grained visual recognition[C] //Proceedings of the European Conference on Computer Vision. Heidelberg: Springer, 2018: 574-589

[6] Wah C, Branson S, Welinder P, et al. The Caltech-UCSD birds-200-2011 dataset[R]. California: California Institute of Technology. Computer Neural System, 2011

[7] Krause J, Stark M, Deng J, et al. 3D object representations for fine-grained categorization[C] //Proceedings of the IEEE International Conference on Computer Vision Workshops. Los Alamitos: IEEE Computer Society Press, 2013: 554-561

[8] Maji S, Rahtu E, Kannala J, et al. Fine-grained visual classification of aircraft[OL]. [2020-05-22]. https://arxiv.org/abs/1306. 5151

[9] Zhang N, Donahue J, Girshick R, et al. Part-based R-CNNs for fine-grained category detection[C] //Proceedings of the European Conference on Computer Vision. Heidelberg: Springer, 2014: 834-849

[10] Wei X S, Xie C W, Wu J X, et al. Mask-CNN: localizing parts and selecting descriptors for fine-grained bird species categorization[J]. Pattern Recognition, 2018, 76: 704-714

[11] Zhao Yili, Xu Dan. Joint semantic parts for fine-grained bird images recognition[J]. Journal of Computer-Aided Design \& Computer Graphics, 2018, 30(8): 1522-1529(in Chinese) (赵毅力, 徐丹. 联合语义部件的鸟类图像细粒度识别 $[\mathrm{J}]$. 计算机辅助设计与图形学学报, 2018, 30(8): 1522-1529)

[12] Deng Z J, Hu X W, Zhu L, et al. $\mathrm{R}^{3}$ Net: recurrent residual refinement network for saliency detection[C] //Proceedings of the 
27th International Joint Conference on Artificial Intelligence. California, 2018: 684-690

[13] Kim J H, On K W, Kim J, et al. Hadamard product for low-rank bilinear pooling[OL]. [2020-05-22]. https://arxiv.org/ abs/1610.04325

[14] Tan M, Wang G J, Zhou J, et al. Fine-grained classification via hierarchical bilinear pooling with aggregated slack mask[J]. IEEE Access, 2019, 7: 117944-117953

[15] ORussakovsky O, Deng J, Su H, et al. ImageNet large scale visual recognition challenge $[\mathrm{J}]$. International Journal of Computer Vision, 2015, 115(3): 211-252

[16] Chen M M, Mitra N J, Huang X L, et al. Global contrast based salient region detection[J]. IEEE Transactions on Pattern Analysis and Machine Intelligence, 2015, 37(3): 569-582

[17] Liu W, Rabinovich A, Berg A C. ParseNet: looking wider to see better[OL]. [2020-05-22]. https://arxiv.org/abs/1506.04579

[18] Zhang H, Xu T, Elhoseiny M, et al. SPDA-CNN: unifying semantic part detection and abstraction for fine-grained recognition[C] //Proceedings of the IEEE Conference on Computer Vision and Pattern Recognition. Los Alamitos: IEEE Computer Society Press, 2016: 1143-1152

[19] Liao Q Y, Holewa H, Xu M, et al. Fine-grained categorization by deep part-collaboration convolution net[C] //Proceedings of the International Conference on Digital Image Computing: Techniques and Applications. Los Alamitos: IEEE Computer Society Press, 2018: 1-8

[20] Zheng H L, Fu J L, Mei T, et al. Learning multi-attention convolutional neural network for fine-grained image recognition[C] //Proceedings of the IEEE International Conference on Computer Vision. Los Alamitos: IEEE Computer Society Press,
2017: 5219-5227

[21] Xin Q, Lv T J, Gao H. Random part localization model for fine-grained image classification[C] //Proceedings of the IEEE International Conference on Image Processing. Los Alamitos: IEEE Computer Society Press, 2019: 420-424

[22] Zhang J, Zhang R S, Huang Y P, et al. Unsupervised part mining for fine-grained image classification[OL]. [2020-05-22]. https://arxiv.org/abs/1902.09941

[23] Gao Y, Beijbom O, Zhang N, et al. Compact bilinear pooling[C] //Proceedings of the IEEE Conference on Computer Vision and Pattern Recognition. Los Alamitos: IEEE Computer Society Press, 2016: 317-326

[24] Kong S, Fowlkes C. Low-rank bilinear pooling for fine-grained classification[C] //Proceedings of the IEEE Conference on Computer Vision and Pattern Recognition. Los Alamitos: IEEE Computer Society Press, 2017: 7025-7034

[25] Cui Y, Zhou F, Wang J, et al. Kernel pooling for convolutional neural networks[C] //Proceedings of the IEEE Conference on Computer Vision and Pattern Recognition. Los Alamitos: IEEE Computer Society Press, 2017: 3049-3058

[26] Wei X, Zhang Y, Gong Y H, et al. Grassmann pooling as compact homogeneous bilinear pooling for fine-grained visual classification[C] //Proceedings of the European Conference on Computer Vision. Heidelberg: Springer, 2018: 365-380

[27] Dai X H, Gong S R, Zhong S, et al. Bilinear CNN model for fine-grained classification based on subcategory-similarity measurement[J]. Applied Sciences, 2019, 9(2): 301

[28] Simonyan K, Zisserman A. Very deep convolutional networks for large-scale image recognition[OL]. [2020-05-22]. https:// arxiv.org/abs/1409.1556 